2018-4

\title{
Integrated Free-Form Method for Aerostructural Optimization of Wind Turbine Blades
}

\author{
Ryan Barrett \\ Brigham Young University, ryan.barrett100@gmail.com \\ Andrew Ning \\ Brigham Young University, aning@byu.edu
}

Follow this and additional works at: https://scholarsarchive.byu.edu/facpub

Part of the Mechanical Engineering Commons

\section{Original Publication Citation}

Barrett, R., and Ning, A., "Integrated Free-Form Method for Aerostructural Optimization of Wind Turbine Blades," Wind Energy, Apr. 2018. doi:10.1002/we.2186

\section{BYU ScholarsArchive Citation}

Barrett, Ryan and Ning, Andrew, "Integrated Free-Form Method for Aerostructural Optimization of Wind Turbine Blades" (2018). Faculty Publications. 2084.

https://scholarsarchive.byu.edu/facpub/2084

This Peer-Reviewed Article is brought to you for free and open access by BYU ScholarsArchive. It has been accepted for inclusion in Faculty Publications by an authorized administrator of BYU ScholarsArchive. For more information, please contact ellen_amatangelo@byu.edu. 


\title{
RESEARCH ARTICLE
}

\section{Integrated Free-Form Method for Aerostructural Optimization of Wind Turbine Blades}

\author{
R. Barrett and A. Ning \\ Brigham Young University, Provo, Utah 84602, USA
}

\begin{abstract}
A typical approach to optimize wind turbine blades separates the airfoil shape design from the blade planform design. This approach is sequential, where the airfoils along the blade span are pre-selected or optimized and then held constant during the blade planform optimization. In contrast, integrated blade design optimizes the airfoils and the blade planform concurrently and thereby has the potential to reduce cost of energy (COE) more than sequential design. Nevertheless, sequential design is commonly performed because of the ease of precomputation, or the ability to compute the airfoil analyses prior to the blade optimization. This research compares two integrated blade design approaches. The precomputational method combines precomputation with the ability to change the airfoil shapes in limited ways during the optimization. The free-form method allows for a complete range of airfoil shapes, but without precomputation. The airfoils are analyzed with a panel method (XFOIL) and a Reynolds-averaged Navier-Stokes computational fluid dynamics method (RANS CFD). Optimizing the NREL 5-MW reference turbine showed COE reductions of 2.0\%, $4.2 \%$, and $4.7 \%$ when using XFOIL and $2.7 \%, 6.0 \%$, and $6.7 \%$ when using RANS CFD for the sequential, precomputational, and free-form methods, respectively. The precomputational method captures the majority of the benefit of integrated design for minimal additional computational cost and complexity, but the free-form method provides modest additional benefits if the extra effort is made in computational cost and development time. Copyright (c) 2016 John Wiley \& Sons, Ltd.
\end{abstract}

\section{KEYWORDS}

turbine blade optimization; integrated blade design; free-form; precomputational; computational fluid dynamics (CFD)

Correspondence

A. Ning, Brigham Young University, 435 CTB, Provo, Utah 84602, USA.

E-mail: aning@byu.edu

\section{NOMENCLATURE}

\author{
$S_{a f}$ airfoil shape parameters \\ $t / c$ airfoil thickness-to-chord ratio \\ $\alpha$ angle of attack \\ AEP annual energy production \\ $B_{a f}$ blended airfoil family factor \\ CST class-shape-transformation \\ CFD computational fluid dynamics \\ COE cost of energy
}

$A$ Kulfan parameters

NREL National Renewable Energy Laboratory

RANS Reynolds-averaged Navier-Stokes 


\author{
TCC turbine capital costs \\ $c_{d}$ two-dimensional drag coefficient \\ $c_{l}$ two-dimensional lift coefficient
}

\title{
1. INTRODUCTION
}

The performance of a wind turbine, as defined by the cost of energy (COE), is predominately determined from the aerodynamics and structures of its blades. A wind turbine blade is defined by a series of two-dimensional airfoils that constitute its three-dimensional shape. A typical blade design is performed sequentially, where the airfoils are chosen or optimized by the designer a priori and then the blade planform is optimized [1-3]. However, optimal airfoil shapes can be dependent on site conditions, turbine type, and the other blade parameters. Therefore, sequential blade design inherently generates sub-optimal blades because the design space is limited to pre-selected airfoil shapes. Integrated blade design optimizes the airfoils and the blade planform concurrently by adding airfoil shape parameters to the optimization as design variables. Regardless of the field or discipline, integrated design performs better than, or at least equal to, sequential design by taking advantage of the interactions between design variables. Figure 1 shows a comparison of sequential and integrated wind turbine blade designs.

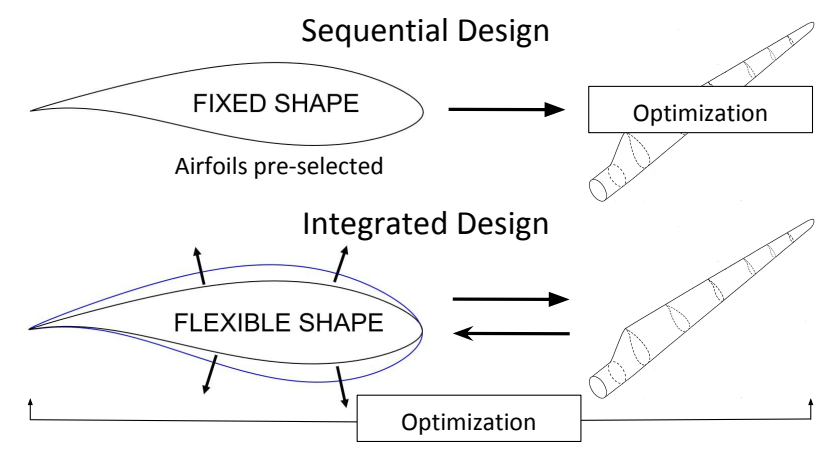

Figure 1. In sequential design the airfoil shapes are pre-selected and held constant and in integrated design the airfoil shapes are allowed to change during the optimization.

Although integrated design has been shown to find better performing blade designs [4-12], one of the main reasons that sequential design is commonly used is for the ability to separate the analysis of the airfoil shapes from the blade optimization, referred to in this paper as precomputation. The aerodynamics and the structures of the blade depend highly on the lift and drag forces along the blade span. These forces are characterized by the lift and drag coefficients that are functions of the airfoil shape, angle of attack, and Reynolds number. During a blade optimization, when design variables such as chord or twist update, the lift and drag coefficients would need to be recomputed at the updated angles of attack. Depending on how the airfoils are analyzed, recomputing at each iteration can be computationally expensive. However, in a typical sequential design the airfoil shapes are pre-selected so that the lift and drag coefficients can be analyzed for a number of angles of attack before the optimization and then read from pretabulated airfoil tables during the optimization.

Our past work explored an integrated design approach that we call the precomputational method because it added the ability to change the airfoil shapes in limited ways, such as by specified airfoil families, while continuing to perform the airfoil analyses prior to the blade optimization [13]. This method shows significant improvements in blade performance for minimal additional computational cost and complexity. The objective of this research is to evaluate the performance of the precomputational method by comparing it to the free-form method, an integrated design approach with no precomputation that allows the airfoils to assume essentially any shape. The workflows of these two integrated design approaches are compared in Figure 2.

The intention of this comparison is to determine if most of the benefit of integrated blade design is captured by the precomputational method or if there is a significant benefit from expanding the range of possible airfoil shapes. The reason that the free-form method is not ideal, despite having a larger airfoil design space, is that precomputation is no longer feasible. Generating a surrogate model that would work with all possible airfoil shapes would require an excessive number of airfoil analyses and thereby negate the benefit of precomputation.

The three main areas to consider with an integrated blade design are the airfoil analysis method (how to obtain the airfoil's lift and drag coefficients), the airfoil parameterization method (how the airfoil coordinates translate into optimization design variables), and the optimization method. 


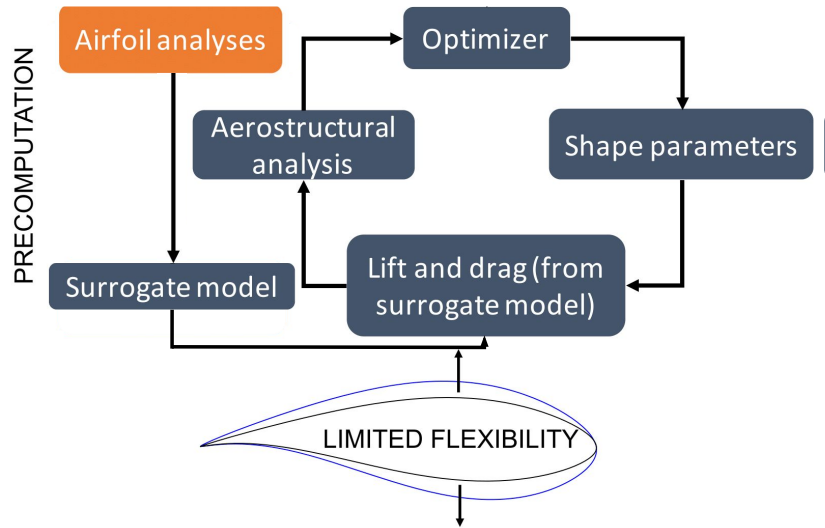

(a) Precomputational method

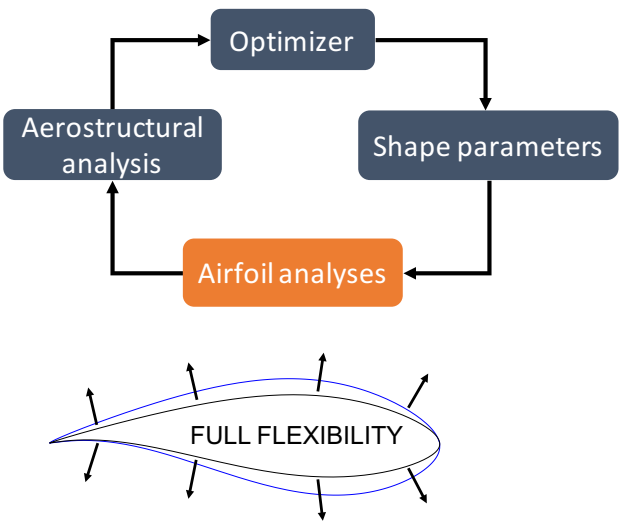

(b) Free-form method

Figure 2. Comparison of workflows for integrated blade design approaches. The precomputational method performs the airfoil analyses and surrogate model generation prior to the blade optimization, while the free-form method performs the airfoil analyses during the blade optimization.

This research extends previous investigations of integrated blade design by using Reynolds-averaged Navier-Stokes computational fluid dynamics (RANS CFD) for the airfoil analysis method and comparing it to a panel method, XFOIL. All known prior integrated designs that use 2D analysis methods have used XFOIL [4-10]; although RANS CFD have been used in 3D CFD integrated design $[11,12]$. While panel methods are relatively fast, they have known problems converging in highly separated flow and are typically not as accurate as other higher-fidelity techniques such as RANS CFD or using a wind tunnel. Barrett et al. compares various airfoil analysis methods of varying fidelity and finds that significant changes in optimal blade design occur [13].

The CST method used in this research for the airfoil parameterization has been highly rated compared to other parameterization methods due to its ability to span the airfoil design space with a relatively low number of parameters and the assurance of a smooth curve $[14,15]$. The method was developed by Kulfan for the purpose of reducing the number of parameters needed to define any $2 \mathrm{D}$ or $3 \mathrm{D}$ smooth aerodynamic shape $[16,17]$ and has successfully been used in aircraft wing design and optimization $[18,19]$.

The choice of optimization method is critical to the feasibility of the free-form method because of the increase of both the number of design variables and the computational cost per function evaluation. Previous research has focused on gradient-free methods [6-9,11] and finite-differencing gradients $[4,5]$ for integrated design. Gradient-free methods in particular are well-suited for finding the global optimum. Vicina et al. demonstrated the potential of gradient-free methods for use with integrated design in wind turbine optimization, even when using CFD [11]. However, because both gradientfree and finite-differencing methods tend to scale poorly with the number of design variables the optimization problems have typically been limited in size. Analytic gradients are well-suited for larger scale problems because they are exact and scale well with the number of design variables. Methods designed for large-scale optimization problems are needed as it is likely that their size will increase as wind turbine optimization continues to develop.

Figure 3 shows the relationship between the number of design variables and the number of function evaluations needed to converge a relatively simple optimization for the different optimization methods of gradient-free, gradientbased finite-differencing, and gradient-based analytic gradient methods for the multidimensional generalization of the Rosenbrock function [20]. Although the Rosenbrock function is less complex than wind turbine optimization, Lyu et al. shows that similar trends exist specifically for aerodynamic shape optimization using RANS CFD [21]. They conclude that gradient-based methods are the only viable option for large-scale aerodynamic design optimization because gradientfree methods can require up to three orders of magnitude more computational effort than gradient-based methods [21]. Rios et al. corroborates this idea when they tested 502 optimization problems with 22 gradient-free solvers and showed that, generally speaking, the performance of the optimizer suffers when there are over 30 design variables [22]. Using RANS CFD with the setup used in this research, the time difference to optimize between using gradient-free, finite-differencing, and analytic gradient methods can be on the order of months, weeks, and days, respectively. There are some downsides to gradient-based methods such as the possibility of converging on a local optimum and the development of the gradients. Nevertheless, these risks can be mitigated through the use of analytic gradients and using a multi-start approach. In many cases local optimum exist due to numerical noise caused by poor gradients. This research uses gradient-based methods to reduce the computational cost and increase the performance of the optimization.

This research contributes to prior investigations of integrated blade design in three main ways: 


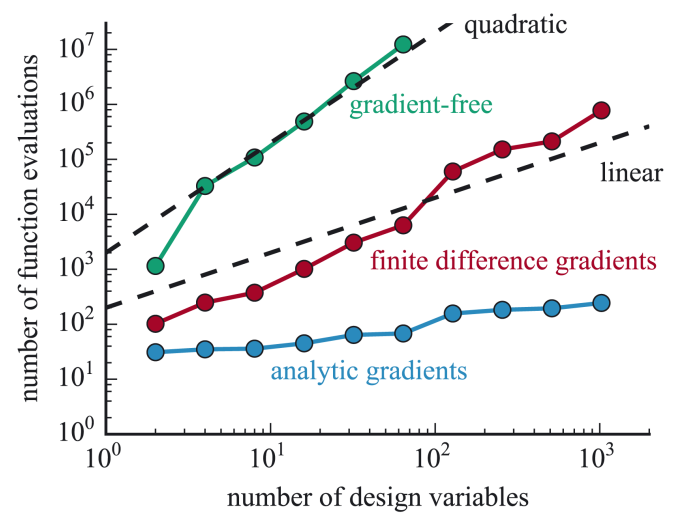

Figure 3. Number of function evaluations required to converge optimization as a function of number of design variables. Sparse Nonlinear OPTimizer is used for the gradient-based results and Augmented Lagrangian Particle Swarm Optimizer for the gradientfree results, however similar trends were observed using Sequential Least SQuares Programming (gradient-based) and Non Sorting Genetic Algorithm II (gradient-free). Reference lines for linear and quadratic scaling are also shown. Image and caption from Ning et al. [20].

- comparing integrated design approaches (free-form and precomputational methods) to sequential design

- comparing XFOIL and RANS CFD airfoil analysis techniques in a free-form approach

- using airfoil shape parameter analytic gradients instead of pure finite-differencing or gradient-free methods

This study contributes to the development of wind turbines by comparing free-form and precomputational integrated design approaches and providing higher-fidelity airfoil analysis techniques for large-scale aerostructural optimization of the cost of energy of wind turbine blades. These methods can be used for more detailed blade design, make wind turbines more cost-effective, and contribute to the world's growing energy needs.

\section{METHODOLOGY}

To generate a practical wind turbine blade design, both the aerodynamics and the structures of the blade must be simultaneously considered in an aerostructural approach. Separating the blade aerodynamic and structural analysis can result in sub-optimal designs that are either too heavy, fail during operation, or have poor energy output.

\subsection{Aerodynamics}

The aerodynamics of the blade are mainly affected by the 2D airfoils' lift and drag coefficients. These coefficients are input into a blade element momentum theory that converts the lift and drag coefficients from all the 2D airfoils into the 3D aerodynamic blade analysis.

\subsubsection{Airfoil Analysis}

The airfoils' lift and drag coefficients have a major effect on the aerostructural analysis. Three common ways to obtain these lift and drag coefficients are through a lower-fidelity panel method (XFOIL), Reynolds-averaged Navier-Stokes computational fluid dynamics (RANS CFD), or using a wind tunnel. The optimal blade shape is sensitive to the airfoil analysis method and therefore the use of higher-fidelity techniques is warranted. Figure 4 shows a comparison of the lift and drag coefficients of an airfoil using XFOIL, RANS CFD, and known wind tunnel data where the trend of the RANS CFD data more closely fits that of the wind tunnel data [23] as compared to XFOIL. The lift and drag coefficients from the aerodynamic analyses in this study are analyzed at a Reynolds number of $10^{6}$ and are rotationally corrected using the NREL AirfoilPreppy Python tool ${ }^{*}$ to prepare for blade analysis. The three-dimensional rotational corrections are performed using Du's method [24] to augment the lift and Eggers' method [25] to modify the drag.

Panel Method (XFOIL) XFOIL is a software program developed by Drela that uses a linear potential (panel) method with an integral boundary layer in the design and analysis of subsonic isolated airfoils [26]. Given the 2D coordinates from

*https://github.com/WISDEM/AirfoilPreppy 


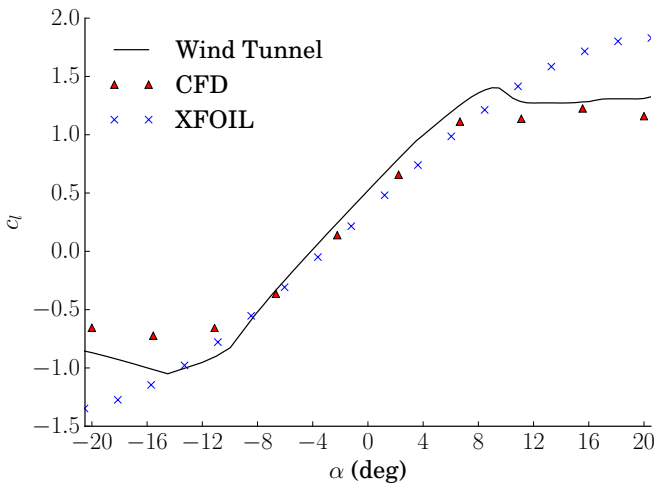

(a) Lift coefficient versus angle of attack

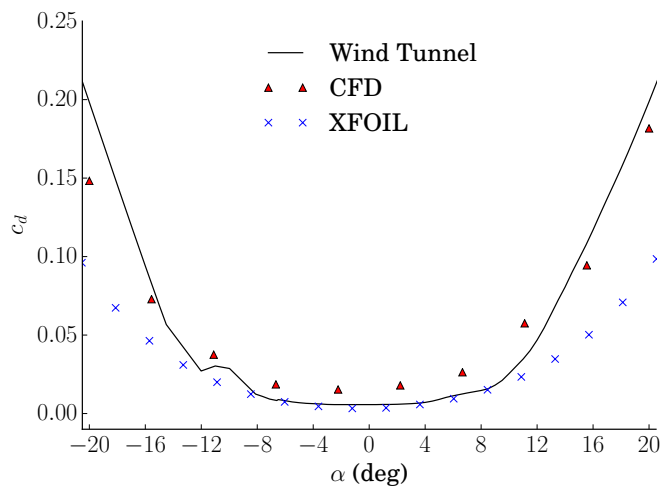

(b) Drag coefficient versus angle of attack

Figure 4. Comparison of different airfoil analysis techniques (XFOIL vs. CFD vs. Wind tunnel) for the DU21_A17 airfoil.

the airfoil parameterization, XFOIL calculates the pressure distribution to obtain the lift and drag coefficients. XFOIL performs the analysis very quickly, but integral boundary layer approaches are not consistent with the physics of highly separated flows and post-stall performance can be inaccurate. Also, since XFOIL is based on idealized computational models, it tends to under-predict drag coefficients and slightly over-predict lift coefficients.

RANS CFD CFD more accurately estimates the airfoil's lift and drag coefficients by better resolving viscous effects, especially post-stall. A comparison of CFD compared to other methods is shown in Figure 4. The open-source CFD software SU2 [27] is used because it provides adjoint gradients and has a Python interface. A major challenge in performing CFD analysis within an optimization is that the mesh needs to be regenerated whenever the airfoil shape changes. The $S U 2$ mesh deformation tool [27] can automatically deform any base mesh to any airfoil shape, a deformed mesh is visualized in Figure 5a and 5b. To calculate the cost of energy for the blade design, fourteen airfoil sections (six airfoil shapes are repeated at different sections) are analyzed along the blade at ten wind speeds to generate the power curve and each airfoil section is also analyzed an additional four times to perform the structural analysis. The 2D airfoil meshes use 14,336 elements in an unstructured C-mesh to balance the trade-off between accuracy and time.

The RANS governing equations use the one-equation Spalart-Allmaras turbulence model with compressible flow. Although other turbulence models such as $k-\omega$ may be more accurate for wind turbine applications [28], this method was chosen because it was the best available in the $S U 2$ CFD package. The convergence criteria specifies a change in residual values of less than $10^{-9}$ with a max number of iterations of 50,000. Using 16 cores, the typical required computational time is several minutes and around 20,000 iterations to convergence. Figure 5c shows the CFD field visualization around one of the airfoils at an angle of attack of zero degrees. For this research, a supercomputer is used to speed up the CFD optimization. However, above about 16 to 32 cores the time savings from additional cores is less than the increase in time from communication overhead between cores. To take advantage of more computing resources, the analysis is setup to run multiple CFD simulations simultaneously.

\subsubsection{Blade Element Momentum Theorem}

Blade element momentum (BEM) theory converts the lift and drag coefficients from all the 2D airfoils into the 3D blade analysis. The chosen BEM method has guaranteed convergence $\left(\mathrm{CCBlade}^{\dagger}\right)$ [29] and is used with the wind blade analysis tool RotorSE ${ }^{\ddagger}$. The BEM theory defines a residual equation (Eq. 1) that must be solved iteratively to converge on the local inflow angle $(\phi)$ [29]. The induction factors $\left(a\right.$ and $\left.a^{\prime}\right)$ are then computed and the lift and drag coefficients of each airfoil section determined at the specified angle of attack.

$$
R(\phi)=\frac{\sin \phi}{1-a(\phi)}-\frac{\cos \phi}{\lambda_{r}\left(1+a^{\prime}(\phi)\right)}=0
$$

Due to the expensive nature of RANS CFD and the iterative behavior of the BEM method [29], the induction factors are converged using lift and drag coefficient splines from XFOIL. The splines are generated from analyzing the airfoil shapes

†https://github.com/WISDEM/CCBlade/

†https://github.com/WISDEM/RotorSE/ 


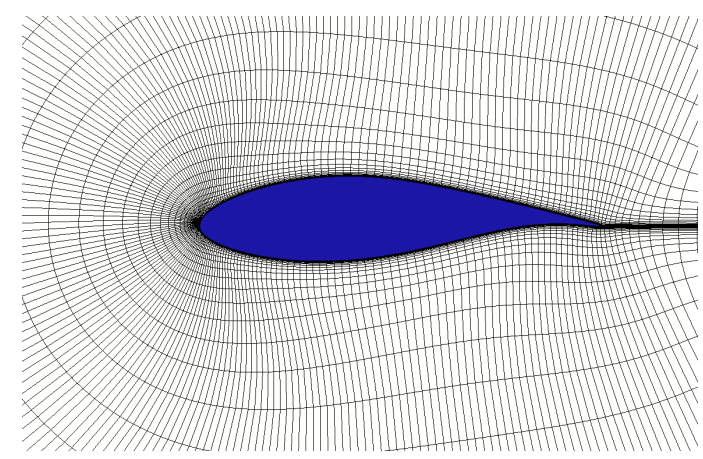

(a) DU21_A17 airfoil mesh near-field

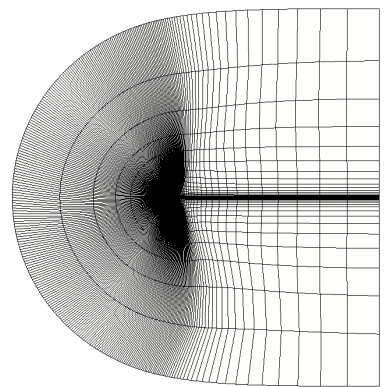

(b) DU21_A17 airfoil C-mesh far-field

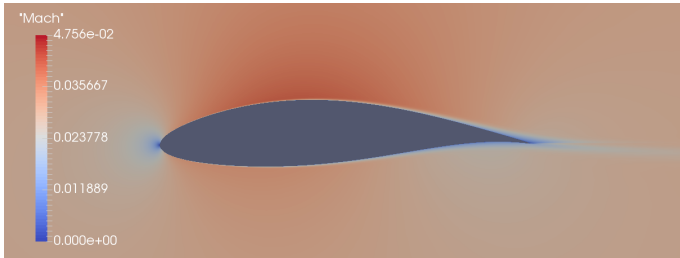

(c) DU21_A17 airfoil Mach number field (compressible flow) at angle of attack of zero degrees

Figure 5. DU21_A17 airfoil unstructured C-mesh created with SU2 mesh deformation tool and CFD field visualization.

at angles of attack from $-30^{\circ}$ to $30^{\circ}$, then the Viterna method is used to extrapolate the lift and drag coefficients from $-180^{\circ}$ to $180^{\circ}$ [30]. Once the induction factors and corresponding angles of attack are known, the specified airfoil analysis method (either XFOIL or RANS CFD) generate the lift and drag coefficients.

\subsection{Structures}

While the blade's aerodynamics are important for calculating the power conversion, the blade's structural analysis is necessary to ensure the blade does not fail during operation. A beam finite element method is used to analyze the blade structure called pBEAM (polynomial beam element analysis module), which uses Euler-Bernoulli beam elements with twelve degrees of freedom (three translational and three rotational at each element end) ${ }^{\S}$ [31]. For the composite materials found along the blade sections, a modified classical lamination theory (CLT) combined with a shear-flow approach is used called PreComp $\uparrow$. Similar structural analysis approaches are fairly common [5,32] and the configuration used is described in more detail by Ning et al. [31]. The airfoil shapes affect the blade structures through the sizing of the sections that, in turn, affect blade mass, strain, etc. Composite panels in the spar cap, web, and trailing edge make up the majority of the structural integrity. There are a number of layers including: the GelCoat, glass fabrics, SNL TRIAX $\left([ \pm 45]_{2}[0]_{2}\right)$, SaerTex Double-Dias (DB, $[ \pm 45]_{4}$ ), carbon fabrics, generic foam, and epoxy resins [31]. These structural composite layers can adapt to changes in the chord and airfoil shapes. Trailing edge material thickness and spar cap material thickness are the optimization variables and the remaining structural elements scale accordingly.

\subsection{Precomputational Method}

The precomputational method is a type of integrated design that allows the airfoil shapes to change during the blade optimization while still computing the airfoils' lift and drag coefficients beforehand. In a typical sequential design, splines are generated for each airfoil to make the lift and drag coefficients a function of angle of attack. In the precomputational method, 2D splines are generated for each airfoil family to make the lift and drag coefficients a function of both angle of attack and an airfoil shape parameter. In this case, the airfoil's thickness-to-chord ratio $(t / c)$ is chosen as the airfoil shape parameter as shown in Figure 6a so that the airfoils are of the same airfoil family. Aerodynamic performance tends to improve with thinner airfoils while the blade's bending stiffness tends to improve with thicker airfoils. Prior to the blade optimization, the lift and drag coefficients are computed for a number of angles of attack and ten airfoils with different thickness-to-chord ratios ranging from $13 \%$ to $42 \%$ within an airfoil family. A surrogate model is generated from the $2 \mathrm{D}$

$\S_{\text {https: //github.com/WISDEM/pBEAM }}$

Thttps://nwtc.nrel.gov/Precomp 
splines of the lift and drag coefficients based on angle of attack and $t / c$. A smoothing factor is applied to the bivariate spline that resulted in maximum error values of 0.01 and 0.005 for the lift and drag coefficients splines, respectively. Surrogate models closely emulate the behavior of the more complicated analysis method, while being computationally inexpensive to evaluate compared to the real analysis method. The lift and drag coefficients can then be easily generated from the surrogate model for an airfoil of any $t / c$ within the specified airfoil family.

The airfoil shape is also given additional flexibility by utilizing a blended airfoil family factor $\left(B_{a f}\right)$ that blends two airfoil families at the same $t / c$ as seen in Figure $6 \mathrm{~b}$. The lift and drag coefficients and the airfoil coordinates are linearly blended based on a factor that varies continuously between 0.0 and 1.0 that indicates the degree to which the second airfoil family is blended into the first. For example, a blend factor of 0.3 indicates that the airfoil is a blend of $70 \%$ of the first airfoil family and $30 \%$ of the second airfoil family. Performing this type of airfoil blending is common practice as some analysis tools, such as FAST [33] through the AirfoilPrep tool, use a similar airfoil blending between sections. This research applies the blending instead to each airfoil section rather than between sections. The airfoil families chosen for this research match those used in the NREL 5-MW reference turbine: the TU Delft and NACA 64-series airfoil families. For this research, the finally obtained solution from the precomputational method is run again with the free-form method to directly compare the performance. Barrett et al. describes the implementation of the precomputational method in more detail [13].

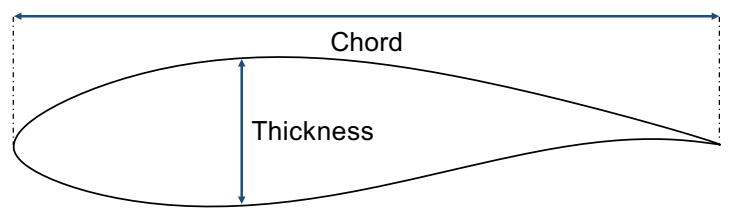

(a) Thickness-to-chord ratio $=$ thickness $/$ chord $=t / c$.

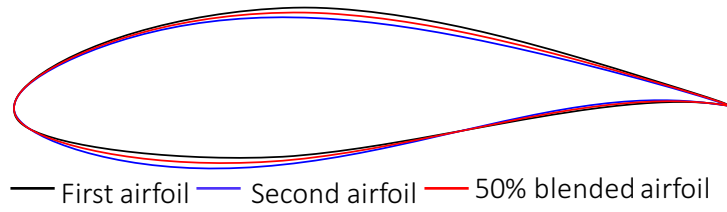

(b) Comparison of $50 \%$ blended airfoil of $t / c$ at $21 \%$.

Figure 6. The airfoil shape parameters used for the precomputational method, thickness-to-chord ratio and blend factor.

\subsection{Free-Form Method}

While there are several methods to parametrize a 2D airfoil shape, including Hicks-Henne bumps, PARSEC technique, and B-spline curves, the CST method is used in this research due to its simplicity, robustness, and the flexibility for a relatively low number of design parameters [15-17,34]. The CST method uses weighting factors, known as Kulfan parameters $\left(A_{1}, \ldots, A_{n}\right)$, to define polynomials that when summed define the top and bottom airfoil surfaces as seen in Figure 7 . For this research, we limit the output coordinates to round nose and pointed aft end airfoils [16].

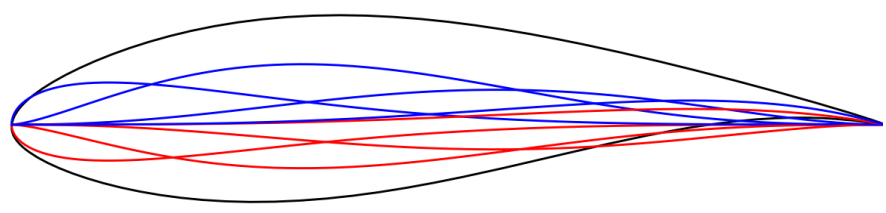

Top surface components ——Bottom surface components

Figure 7. The DU21_A17 airfoil is defined by the summation of weighted polynomial components (four on top and four on bottom).

Based on previous uses of the CST method in optimization and to minimize the number of additional design variables, a third-order Bernstein polynomial with four Kulfan parameters defines both the top and bottom airfoil surfaces [17]. To match the NREL 5-MW reference turbine, six airfoils are used for a total of 48 additional design variables (six airfoils each with eight Kulfan parameters).

\subsection{Airfoil Shape Gradients}

Obtaining analytic gradients can substantially reduce the computational cost due to fewer function calls and faster convergence. A convenient way to obtain gradients is to split the analysis into smaller sections and then combine the partial derivatives of each section using a coupled adjoint solve to obtain total system derivatives [35]. Many of the 
analytic gradients in the blade analysis code, RotorSE, have already been developed using a combination of automatic differentiation and the adjoint method [20]. For this research, we add the gradients for the airfoil shape parameters $\left(S_{a f}\right)$. The equation for the total load gradients is found through a combination of partial derivatives shown in Eq. 2.

$$
\frac{\text { dloads }}{d S_{a f}}=\frac{\partial \text { loads }}{\partial S_{a f}}-\frac{\partial \text { loads }}{\partial \phi} \frac{\partial R}{\partial S_{a f}} / \frac{\partial R}{\partial \phi}
$$

Where $R$ is the residual equation from the BEM method (Eq. 1), $\phi$ is the local inflow angle, and $S_{a f}$ are the airfoil shape parameters. $\partial R / \partial \phi$ is obtained using the Tapenade automatic differentiation tool [36]. The values for $\partial l o a d s / \partial S_{a f}$ and $\partial R / \partial S_{a f}$ are dependent on the gradients of lift and drag coefficients with respect to the airfoil shape parameters $\left(\partial c_{l} / \partial S_{a f}\right.$ and $\left.\partial c_{d} / \partial S_{a f}\right)$. In the case when XFOIL is the airfoil analysis method, complex step is used to find $\partial c_{l} / \partial S_{a f}$ and $\partial c_{d} / \partial S_{a f}$ by using a version of XFOIL that can handle complex numbers". Complex step is similar to finitedifferencing except that the gradients are exact due to the use of complex numbers that avoid the subtraction cancellation error.

For CFD, it is more complicated to obtain $\partial c_{l} / \partial S_{a f}$ and $\partial c_{d} / \partial S_{a f}$. Surface sensitivities for the lift and drag coefficients at the airfoil coordinates normal to the airfoil surface $\left(\partial c_{l} / \partial x_{1}, \ldots, \partial c_{l} / \partial x_{m}\right)$ are generated from $S U 2$ 's automatic differentiation tool [37,38]. The direction of the unit normal vector of a point on the surface is the average of the normal vectors of the lines to its adjacent points. An example of these surface sensitivities on an airfoil is shown in Figure 8. These surface sensitivities help the optimizer to determine what effect a change in the airfoil shape at that airfoil coordinate will have on that airfoil's lift and drag coefficient.

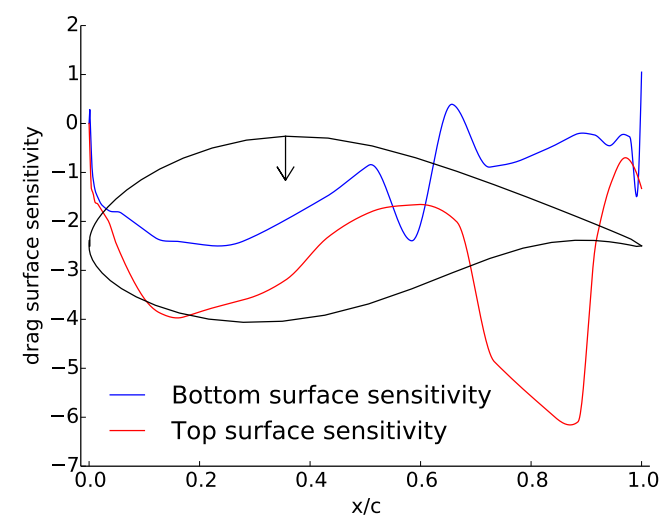

Figure 8. Surface drag coefficient sensitivities $\left(\partial c_{d} / \partial x_{1}, \ldots, \partial c_{d} / \partial x_{m}\right)$ for each airfoil coordinate normal to the surface of the DU21_A17 airfoil and used to find $\partial c_{d} / \partial S_{a f}$.

The chain rule is used in Eq. 3 to convert the lift and drag coefficient gradients with respect to each airfoil coordinate to the lift and drag coefficient gradients with respect to each Kulfan parameter in the CST method [17]. The gradients of the Kulfan parameters with respect to each airfoil coordinate $\left(\partial x_{1} / \partial A_{1}, \ldots, \partial x_{m} / \partial A_{n}\right)$ are found using automatic differentiation of the CST equations.

$$
\frac{\partial c_{l}}{\partial S_{a f}}=\left[\begin{array}{c}
\frac{\partial c_{l}}{\partial A_{1}} \\
\frac{\partial c_{l}}{\partial A_{2}} \\
\vdots \\
\frac{\partial c_{l}}{\partial A_{n}}
\end{array}\right]=\left[\begin{array}{ccc}
\frac{\partial x_{1}}{\partial A_{1}} & \cdots & \frac{\partial x_{m}}{\partial A_{1}} \\
\frac{\partial x_{1}}{\partial A_{2}} & \cdots & \frac{\partial x_{m}}{\partial A_{2}} \\
\vdots & \ddots & \vdots \\
\frac{\partial x_{1}}{\partial A_{n}} & \cdots & \frac{\partial x_{m}}{\partial A_{n}}
\end{array}\right]\left[\begin{array}{c}
\frac{\partial c_{l}}{\partial x_{1}} \\
\frac{\partial c_{l}}{\partial x_{2}} \\
\vdots \\
\frac{\partial c_{l}}{\partial x_{m}}
\end{array}\right]
$$

The values for $\partial c_{l} / \partial S_{a f}$ and $\partial c_{d} / \partial S_{a f}$ are used to obtain the total loads gradients with respect to the airfoil shape parameters (dloads $/ d S_{a f}$ ) from Eq. 2 . The load gradients are propagated to the objective function and the constraints gradients using OpenMDAO [39], which uses either the direct method or the adjoint method depending on the number of inputs to outputs.

\subsubsection{Optimization Problem Setup}

The optimization objective is to minimize the cost of energy, the total cost of the turbine divided by its energy production. The analysis uses a simplified model that assumes that the other aspects of the turbine (hub, nacelle, and tower) remain

Inttps://bitbucket.org/mdolab/pyxlight 
constant because the rotor thrust is constrained to not exceed its initial thrust and the rated power is held constant. In this case, financing aspects are ignored and the cost of energy is found with Eq. 4 [40].

$$
C O E=\frac{F R(T C C+B O S)+(1-T) O P E X}{A E P}
$$

In this equation, $\mathrm{COE}$ is the project levelized cost of energy, TCC is the total turbine capital costs for the project, BOS is the total balance of station costs for the project, AEP is the annual energy production, OPEX is the overall project operational expenditures, FR is the financing rate, and $\mathrm{T}$ is the tax deduction rate on OPEX. The TCC is the sum of the cost of the tower, nacelle, and rotor. In this case, we assume the tower cost and the nacelle cost remain constant. Rotor cost is the sum of the hub cost and the blades cost where the blades costs are estimated to be linearly proportional to the blade mass. An AEP loss factor of 0.885 and the turbine capital cost multiplier of 1.56 are used with a FR of 0.095 and $\mathrm{T}$ of 0.4 [40]. Standard International Electrotechnical Commission (IEC) specifications for a land-based high-wind-speed site (IEC Class IB) are used corresponding to a mean wind speed of $10.0 \mathrm{~m} / \mathrm{s}$ [41]. The wind conditions follow a Weibull distribution with a shape parameter of 2.0. The relevant design variables in each design scenario are summarized in Table I and explained in more detail in previous research [13,31].

Table I. Number of Design Variables Summary

\begin{tabular}{llccc}
\hline Design Variable & & Sequential & Precomputational & Free-Form \\
\hline chord distribution & $c$ & 4 & 4 & 4 \\
max chord location & $c_{\max }$ & 1 & 1 & 1 \\
twist distribution & $\theta$ & 4 & 4 & 4 \\
tip-speed ratio in Region 2 & $\lambda_{2}$ & 1 & 1 & 1 \\
trailing edge material thickness distribution & $t_{t e}$ & 5 & 5 & 5 \\
spar cap material thickness distribution & $t_{\text {spar }}$ & 5 & 5 & 5 \\
thickness-to-chord ratio distribution & $t / c$ & - & 6 & - \\
blended airfoil family factor & $B_{a f}$ & - & 6 & - \\
Kulfan parameters (CST) & $A$ & - & - & 48 \\
\hline total \# & & 20 & 32 & 68 \\
\hline
\end{tabular}

Structural constraints ensure that the blade does not fail during operation. The max strain and buckling in the spar cap and trailing edge are constrained for extreme load and maximum operating conditions. The blade natural frequencies are constrained to be above the rotor rotation speed with an added margin to avoid resonance. The rotor thrust is constrained to not exceed its initial thrust and the rated power is kept constant at 5-MW to ensure that the same tower and drivetrain can be used. In total, there are 33 constraints on the optimization that are described in more detailed equations by Ning et al. [31].

A gradient-based sequential quadratic programming method referred to as the Sparse Nonlinear OPTimizer (SNOPT) [42] is used for the optimization. The formulation of the optimization is summarized below:

$$
\begin{array}{ll}
\text { minimize } & C O E(x) \\
\text { with respect to } & \left.x=S_{a f} \text { (either } t / c \text { and } B_{a f}, \text { or } A\right), c, c_{\text {max }}, \theta, \lambda_{2}, t_{s p a r}, t_{t e} \\
\text { subject to } & c_{\text {set }}(x)<0 \text { (buckling, strain, frequency, rotor thrust) }
\end{array}
$$

Since there are 34 outputs ( 33 constraints and one objective), the sequential and precomputational methods use the direct method within the OpenMDAO [39] framework because the number of design variables is fewer than the number of outputs at 20 and 32 respectively while the free-form method uses the adjoint method because the number of design variables is 68 . The design variables are scaled so that the gradients are of a similar magnitude and the initial airfoil shapes and blade parameters are taken from the NREL 5-MW reference turbine [23]. 


\section{RESULTS}

The main results from the optimization of the NREL 5-MW reference turbine are summarized in Table II** that shows a comparison of the COE, AEP, and TCC in each optimization case grouped by the airfoil analysis method (either XFOIL or RANS CFD) and Figure 9 that shows the percent changes graphically. Results show that the integrated designs had significant reductions in COE over the sequential design. For XFOIL, the COE reduction was 2.0\%, $4.2 \%$, and $4.7 \%$ for the sequential, precomputational, and free-form designs, respectively. For RANS CFD, the COE reduction was $2.7 \%$, $6.0 \%$, and $6.7 \%$ for the sequential, precomputational, and free-form methods, respectively. As expected, additional airfoil shape flexibility results in a larger COE reduction, mainly due to an increase in energy production rather than a reduction in blade mass and thus turbine cost.

Table II. Optimization Main Results Summary

\begin{tabular}{l|l|r|r|r|r} 
& & Reference & Sequential & Precomputational & Free-Form \\
\hline XFOIL & COE (cents/kWh) & 6.283 & $6.155(-2.0 \%)$ & $6.020(-4.2 \%)$ & $5.990(-4.7 \%)$ \\
& AEP $($ MWh $)$ & 23,232 & $23,529(+1.3 \%)$ & $23,932(+3.0 \%)$ & $24,176(+4.1 \%)$ \\
& TCC $(\$)$ & $9,207,436$ & $9,100,633(-1.2 \%)$ & $9,029,051(-1.9 \%)$ & $9,098,397(-1.2 \%)$ \\
\hline \multirow{2}{*}{ RANS CFD } & COE (cents/kWh) & 7.512 & $7.311(-2.7 \%)$ & $7.058(-6.0 \%)$ & $7.002(-6.7 \%)$ \\
& AEP $($ MWh) & 19,433 & $19,872(+2.3 \%)$ & $20,524(+5.6 \%)$ & $20,835(+7.2 \%)$ \\
& TCC $(\$)$ & $9,207,436$ & $9,143,561(-0.7 \%)$ & $9,103,081(-1.1 \%)$ & $9,198,973(-0.1 \%)$
\end{tabular}

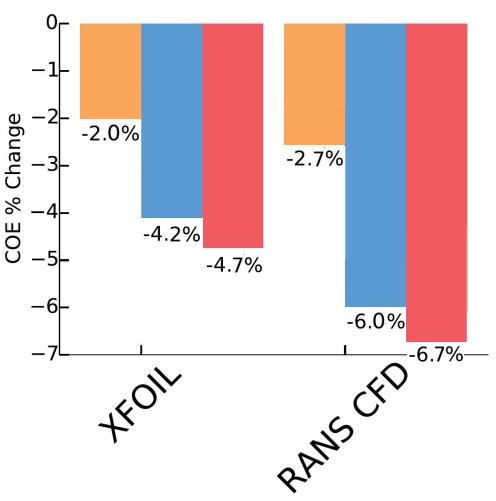

(a) $\mathrm{COE}$

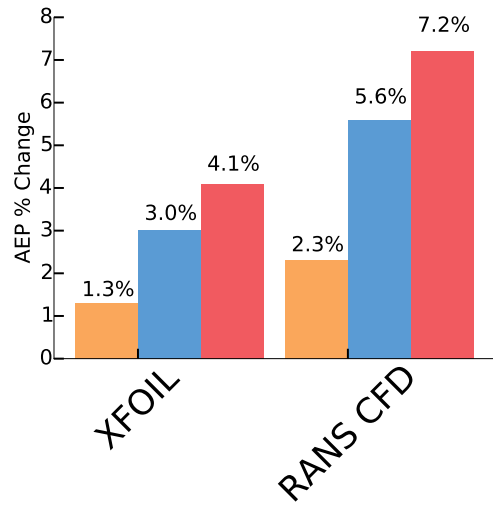

(b) AEP

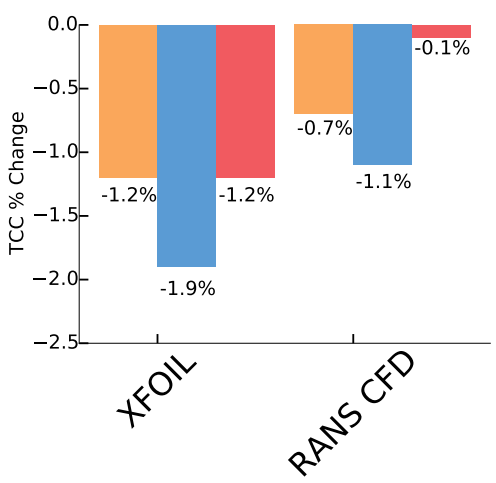

(c) TCC

sequential

free-form

Figure 9. Summary of main results for sequential, precomputational, and free-form methods using XFOIL or RANS CFD. The precomputational method achieves the majority of the performance improvement, but the free-form method achieves some additional benefits.

A comparison of the chord, twist, spar cap thickness, and trailing edge thickness for each optimization case along the blade span is shown in Figure 10. A bigger change occurred, on average, by switching the airfoil analysis method from XFOIL to RANS CFD than by changing the airfoil shape parameters. The blades when analyzed with RANS CFD tended to have larger chord, more twist, and thicker materials than those from using XFOIL. This is because, in general, the drag coefficients were higher for RANS CFD than XFOIL and therefore the blade was forced to have a larger size to withstand the increased loads. When comparing the blade parameters between the precomputational and free-form methods, the freeform method also tended to have heavier properties (i.e., larger chord, thickness material distributions, etc.). The free-form method was able to generate airfoil shapes that had higher overall loads that resulted in increased energy production, but these increased loads had to be balanced with a larger blade. The change in chord, twist, etc. between the different design methods show that the optimal blade planform is dependent on the airfoils and vice versa.

A graphical representation of the airfoil shapes cross-sections taken at different points along the blade span is shown in Figure 11, where Airfoil 1 starts near the blade root and progresses to Airfoil 6 near the blade tip. The airfoil shape

** The complete optimization results for each optimization case can be found at http://flow.byu.edu/publications. 
changes, in most cases, were relatively subtle, but still had a large impact on COE. The optimal airfoil shapes changed more from the original NREL 5-MW reference turbine airfoils with the RANS CFD cases than the XFOIL cases. This is why the benefit of integrated design was greater for RANS CFD, because the original airfoils when evaluated using RAN CFD were worse to begin with and the integrated design was thereby able to extract a greater benefit. In many cases, the airfoil shapes changes between the precomputational and the free-form methods were relatively similar. This shows that the precomputational method can converge on similar airfoil shapes despite having fewer parameters.

Results show that the integrated free-form method reduced COE by $2.7 \%$ and $4.0 \%$ more than the sequential design and $0.5 \%$ and $0.7 \%$ more than integrated precomputational method when using XFOIL and RANS CFD, respectfully. Precomputational methods can capture the majority of the benefit of integrated design for minimal additional computational cost and complexity. The precomputational method captured $81.5 \%$ and $82.5 \%$ of the benefit found by the free-form method over the sequential design for XFOIL and RANS CFD, respectively. These results are promising for the adoption of the precomputational method because it was able to capture the majority of the benefit of integrated design (about $80 \%$ ) without sacrificing the ease of precomputation. In many situations, the additional cost and complexity of the free-form method for only about $20 \%$ additional benefit may not be worth it. By carefully choosing the airfoil shape parameters, many of the major changes in the airfoil shapes can be obtained using fewer parameters. The choice between the two integrated design approaches balance the trade-off between computational cost and performance. If the extra effort is made, in both development time and computational cost, to obtain analytic gradients and perform the airfoil analyses during the optimization, the free-form method can reduce the COE more than the precomputational method. As wind energy continues to advance and computational capabilities increase, the extra benefit from a free-form method may be worth the additional cost and effort.

\section{CONCLUSIONS}

Integrated blade design optimizes the airfoils and the blade planform concurrently, but because the airfoil shapes change during the optimization one major challenge is in analyzing the airfoils' lift and drag coefficients. The precomputational method has been developed to allow the airfoil shapes to change during the optimization while still performing the airfoil analyses prior to the optimization. This research compares the precomputational method to the free-form method, an integrated design method that allows for a complete range of airfoils shapes without precomputation. The objective of this comparison is to determine how much of the potential performance of integrated design can be captured by the precomputational method. This study quantified the difference in COE reduction between the integrated and sequential design approaches. Results showed that the integrated free-form method reduced COE by $2.7 \%$ and $4.0 \%$ more than the sequential design and $0.5 \%$ and $0.7 \%$ more than the integrated precomputational method when using XFOIL and RANS CFD, respectfully. The precomputational method captured $81.5 \%$ and $82.5 \%$ of the benefit found by the free-form method over the sequential design for XFOIL and RANS CFD, respectively. Precomputational methods can capture the majority of the benefit (about $80 \%$ in this case) of integrated design for minimal additional computational cost and complexity. By maintaining the ease of precomputation, this method has a similar workflow to the sequential design, but manages to add some airfoil shape flexibility.

The free-form method was able to achieve an additional $25.0 \%$ and $21.2 \%$ improvement over the precomputational method as a result of the additional airfoil shape flexibility for XFOIL and RANS CFD, respectively. This improvement comes through the additional effort in both development time and computational cost to obtain analytic gradients and perform the airfoil analyses during the optimization. The trade-off between the precomputational and free-form methods is between computational cost and improved blade performance.

The importance of using higher-fidelity airfoil analysis methods, such as RANS CFD, has been demonstrated by this study. The airfoil analysis method plays a large role on the optimal blade planform while the airfoil parameterization method has a more moderate influence. Through techniques developed in this study, such as though the use of analytic gradients, higher-fidelity analysis methods can be used for both the precomputational and the free-form methods so that blade designs can more accurately model real wind turbine blade behavior. This study optimized the NREL 5-MW reference turbine, but similar behavior is expected from integrated blade design for other turbine types and site conditions. The code is planned to be added to the open-source NREL WISDEM wind turbine analysis framework ${ }^{\dagger \dagger}$.

Future work is needed to continue to investigate integrated blade design. Similar to how we increased the flexibility of the airfoil shapes, additional freedom could be given to the structural design through the choice of materials in the composite layers, the thicknesses of each individual layup instead of the entire composite, or the ply orientation and order. Providing the structural design variables will likely provide additional benefits in reducing COE.

\footnotetext{
${ }^{\dagger \dagger}$ https://github.com/WISDEM/
} 


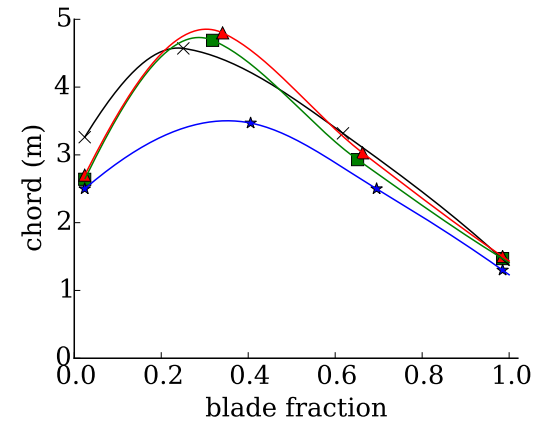

(a) XFOIL - Chord distribution

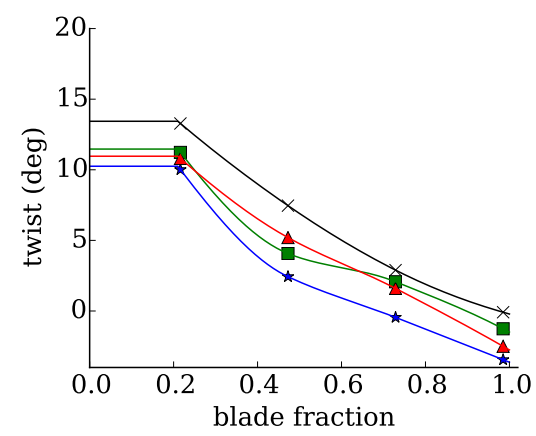

(c) XFOIL - Twist distribution

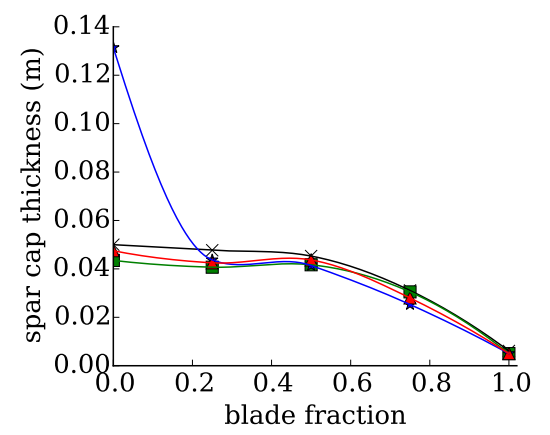

(e) XFOIL - Spar cap thickness distribution

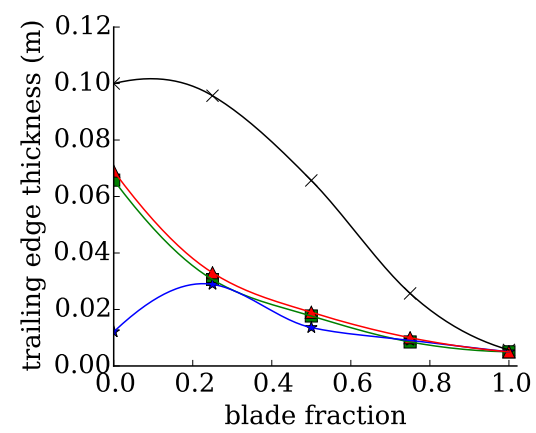

(g) XFOIL - Trailing edge thickness distribution reference $\square$ sequential

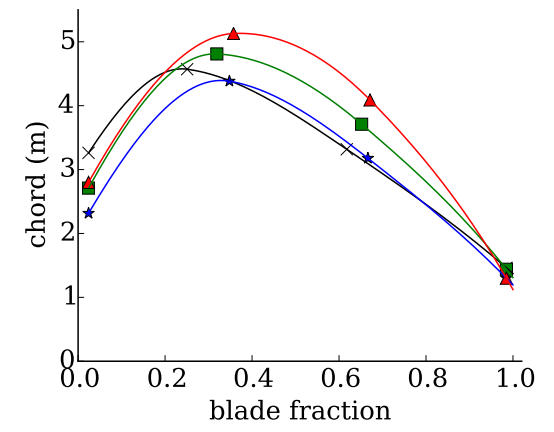

(b) CFD - Chord distribution

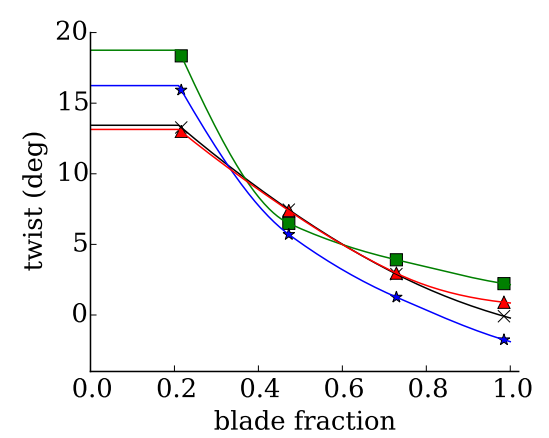

(d) CFD - Twist distribution

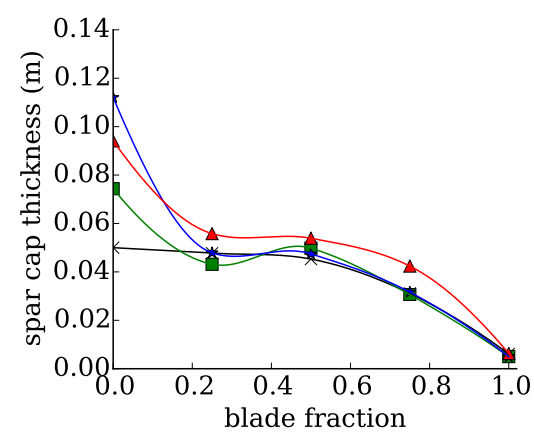

(f) CFD - Spar cap thickness distribution

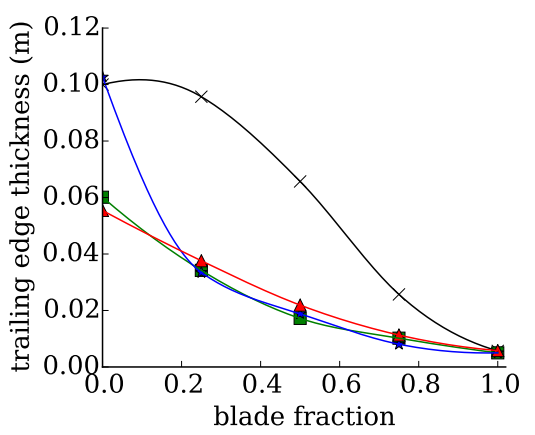

(h) CFD - Trailing edge thickness distribution $\star \star$ precomputational $\Delta \Delta$ free-form

Figure 10. Chord, twist, and spar cap and trailing edge material distributions. 

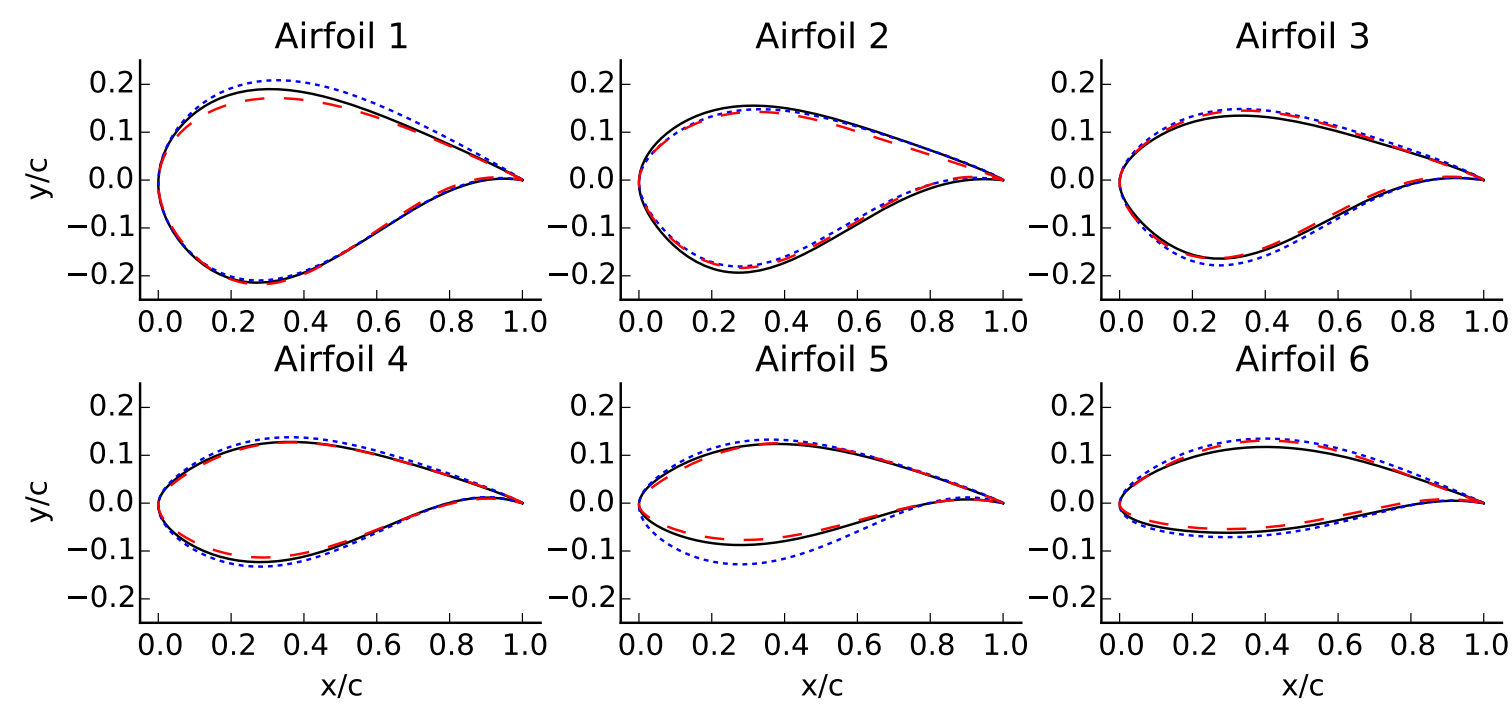

(a) XFOIL
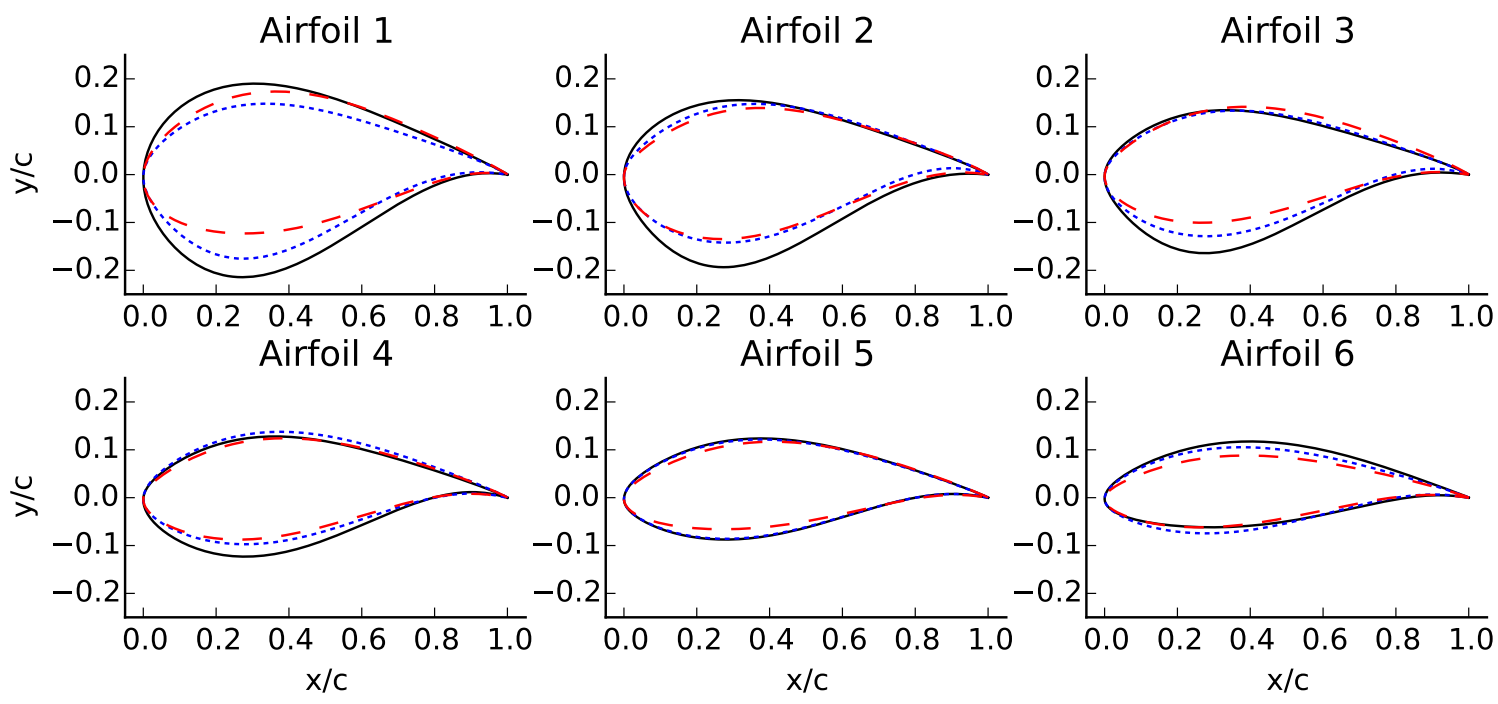

(b) RANS CFD

\section{- reference …. precomputational - - free-form}

Figure 11. Airfoil shape comparison results. 


\section{REFERENCES}

1. Chehouri A, Younes R, Ilinca A, Perron J. Review of performance optimization techniques applied to wind turbines. Applied Energy 2015; 142:361-388, doi:10.1016/j.apenergy.2014.12.043.

2. Xudong W, Shen WZ, Zhu WJ, Sørensen JN, Jin C. Shape optimization of wind turbine blades. Wind Energy 2009; 12(8):781-803, doi:10.1002/we.335.

3. Méndez J, Greiner D. Wind blade chord and twist angle optimization by using genetic algorithms. Proceedings of the Fifth International Conference on Engineering Computational Technology, vol. 6, 2006; 12-15.

4. Bottasso C, Croce A, Sartori L, Grasso F. Free-form design of rotor blades. Journal of Physics: Conference Series, vol. 524, IOP Publishing, 2014; 012 041, doi:10.1088/1742-6596/524/1/012041.

5. Kenway G, Martins J. Aerostructural shape optimization of wind turbine blades considering site-specific winds. Proceedings of the 12th AIAA/ISSMO Multidisciplinary Analysis and Optimization Conference, Sept, 2008; 10-12, doi:10.2514/6.2008-6025.

6. Polat O, Tuncer IH. Aerodynamic shape optimization of wind turbine blades using a parallel genetic algorithm. Procedia Engineering 2013; 61:28 - 31, doi:10.1016/j.proeng.2013.07.088.

7. Herbert-Acero JF, Martínez-Lauranchet J, Probst O, Méndez-Díaz S, Valenzuela-Rendón M, Réthoré PE, CastilloVillar KK. Aerodynamic optimization of small wind turbine rotors based on NACA 4-digit airfoils through computational intelligence. European Wind Energy Conference and Exhibition, 2014; 1-10.

8. Fiore G, Selig MS. Optimization of wind turbine airfoils subject to particle erosion. 33rd AIAA Applied Aerodynamics Conference, 2015; 3393, doi:10.2514/6.2015-3393.

9. Fischer GR, Kipouros T, Savill AM. Multi-objective optimisation of horizontal axis wind turbine structure and energy production using aerofoil and blade properties as design variables. Renewable Energy 2014; 62:506 - 515, doi: 10.1016/j.renene.2013.08.009.

10. Zhu WJ, Shen WZ, Sørensen JN. Integrated airfoil and blade design method for large wind turbines. Renewable Energy 2014; 70:172-183.

11. Vucina D, Marinic-Kragic I, Milas Z. Numerical models for robust shape optimization of wind turbine blades. Renewable Energy 2016; 87, Part 2:849 - 862, doi:10.1016/j.renene.2015.10.040.

12. Dhert T, Ashuri T, Martins JRRA. Aerodynamic shape optimization of wind turbine blades using a Reynolds-averaged Navier-Stokes model and an adjoint method. Wind Energy 2016; doi:10.1002/we.2070.

13. Barrett R, Ning A. Comparison of airfoil precomputational analysis methods for optimization of wind turbine blades. IEEE Transactions on Sustainable Energy Jul 2016; 7(3):1081-1088, doi:10.1109/TSTE.2016.2522381.

14. Kulfan BM, Bussoletti JE. Fundamental parametric geometry representations for aircraft component shapes. 11th AIAA/ISSMO multidisciplinary analysis and optimization conference, vol. 6948, 2006, doi:10.2514/6.2006-6948.

15. Sripawadkul V, Padulo M, Guenov M. A comparison of airfoil shape parameterization techniques for early design optimization. 13th AIAA/ISSMO multidisciplinary analysis optimization conference, 2010; 13-15.

16. Kulfan B. CST universal parametric geometry representation method with applications to supersonic aircraft. 4th International Conference on Flow Dynamics, 2007; 26-28.

17. Hewitt P, Marques S. Aerofoil optimisation using CST parameterisation in SU2. Royal Aeronautical Society Biennial Applied Aerodynamics Research Conference, 2014.

18. Straathof MH, L Van Tooren MJ. Extension to the class-shape-transformation method based on B-splines. AIAA journal 2011; 49(4):780-790.

19. Ciampa PD, Zill T, Nagel B. CST parametrization for unconventional aircraft design optimization. 27th Congress of the International Council of the Aeronautical Sciences (ICAS), 2010.

20. Ning A, Petch D. Integrated design of downwind land-based wind turbines using analytic gradients. Wind Energy Dec 2016; 19(12):2137-2152, doi:10.1002/we.1972.

21. Lyu Z, Xu Z, Martins J. Benchmarking optimization algorithms for wing aerodynamic design optimization. Proceedings of the 8th International Conference on Computational Fluid Dynamics, Chengdu, Sichuan, China, 2014.

22. Rios LM, Sahinidis NV. Derivative-free optimization: a review of algorithms and comparison of software implementations. Journal of Global Optimization Jul 2013; 56(3):1247-1293, doi:10.1007/s 10898-012-9951-y.

23. Jonkman JM, Butterfield S, Musial W, Scott G. Definition of a 5-MW reference wind turbine for offshore system development. National Renewable Energy Laboratory Golden, CO, 2009.

24. Du Z, Selig MS. A 3-D stall-delay model for horizontal axis wind turbine performance prediction. AIAA Paper 1998; 21.

25. Eggers A, Chaney K, Digumarthi R. An assessment of approximate modeling of aerodynamic loads on the UAE rotor. ASME 2003 Wind Energy Symposium, American Society of Mechanical Engineers, 2003; 283-292.

26. Drela M. XFOIL: An analysis and design system for low Reynolds number airfoils. Low Reynolds Number Aerodynamics, Lecture Notes in Engineering, vol. 54, Mueller T (ed.). Springer Berlin Heidelberg, 1989; 1-12, doi:10.1007/978-3-642-84010-4. 
27. Palacios F, Colonno MR, Aranake AC, Campos A, Copeland SR, Economon TD, Lonkar AK, Lukaczyk TW, Taylor TW, Alonso JJ. Stanford University Unstructured (SU2): An open-source integrated computational environment for multi-physics simulation and design. AIAA Paper 2013; 287:2013, doi:10.2514/6.2013-287.

28. Villalpando F, Reggio M, Ilinca A. Assessment of turbulence models for flow simulation around a wind turbine airfoil. Modelling and simulation in engineering 2011; 2011:6, doi:10.1155/2011/714146.

29. Ning A. A simple solution method for the blade element momentum equations with guaranteed convergence. Wind Energy 2014; 17(9):1327-1345, doi:10.1002/we.1636.

30. Viterna LA, Janetzke DC. Theoretical and experimental power from large horizontal-axis wind turbines. Technical Report, National Aeronautics and Space Administration, Cleveland, OH (USA). Lewis Research Center 1982.

31. Ning A, Damiani R, Moriarty PJ. Objectives and constraints for wind turbine optimization. Journal of Solar Energy Engineering June 2014; 136(4):04 010-041 010, doi:10.1115/1.4027693.

32. Kong C, Bang J, Sugiyama Y. Structural investigation of composite wind turbine blade considering various load cases and fatigue life. Energy 2005; 30(11):2101 - 2114, doi:http://dx.doi.org/10.1016/j.energy.2004.08.016.

33. Jonkman JM, Buhl Jr ML. FAST users guide. Technical Report, National Renewable Energy Laboratory 2005.

34. Castonguay P, Nadarajah S. chap. Effect of Shape Parameterization on Aerodynamic Shape Optimization. Aerospace Sciences Meetings, American Institute of Aeronautics and Astronautics, 2007, doi:10.2514/6.2007-59.

35. Martins JR, Hwang JT. Review and unification of methods for computing derivatives of multidisciplinary computational models. AIAA journal 2013; 51(11):2582-2599, doi:10.2514/1.J052184.

36. Hascoet L, Pascual V. The Tapenade Automatic Differentiation tool: principles, model, and specification. ACM Transactions on Mathematical Software (TOMS) 2013; 39(3):20.

37. Jameson A, Shankaran S, Martinelli L. Continuous adjoint method for unstructured grids. AIAA journal 2008; 46(5):1226-1239.

38. Albring T. Gradient calculation using algorithmic differentiation. RAE 2013; 2822:10.

39. Gray JS, Moore KT, Naylor BA. OpenMDAO: An Open Source Framework for Multidisciplinary Analysis and Optimization. 13th AIAA/ISSMO Multidisciplinary Analysis and Optimization Conference, AIAA-2010-9101, AIAA: Fort Worth, Texas, 2010, doi:10.2514/6.2010-9101.

40. Ning A, Dykes K. Understanding the benefits and limitations of increasing maximum rotor tip speed for utility-scale wind turbines. Journal of Physics: Conference Series Jun 2014; 524(012087), doi:10.1088/1742-6596/524/1/012087.

41. European Committee for Standardisation. Eurocode 3: Design of Steel Structures-Part 1-6: General rulesSupplementary rules for the shell structures. EN 1993-1-6: 20xx, European Committee for Standardisation 1993.

42. Gill PE, Murray W, Saunders MA. SNOPT: An SQP algorithm for large-scale constrained optimization. SIAM Journal on Optimization 2002; 12(4):979-1006, doi:10.1137/S0036144504446096. 